\title{
Bandlimited Airy Pulses for Invariant Propagation in Single-Mode Fibers
}

Miguel A. Preciado and Miguel A. Muriel,

\begin{abstract}
By spectral analysis, and using joint time-frequency representations, we present the theoretical basis to design invariant bandlimited Airy pulses with an arbitrary degree of robustness and an arbitrary range of single-mode fiber chromatic dispersion. The numerically simulated examples confirm the theoretically predicted pulse partial invariance in the propagation of the pulse in the fiber.
\end{abstract}

Index Terms-Fiber optics, optical fiber communication, optical fiber dispersion, optical pulse shaping.

\section{INTRODUCTION}

I DEAL Airy pulses are an infinite energy kind of pulse which propagates undistorted on dispersive media, proposed in [1] within the context of quantum mechanics. Due to the unique properties of Airy pulses, the intensity profile remains invariant during propagation. However, ideal Airy pulses are impractical because of their infinite energy. The properties of finite-energy Airy beams by temporal truncation were investigated theoretically within the context of optics in [2]. Over the years, nondiffracting waves have been systematically investigated, and in linear optics, spatial Airy beams have been proposed for several applications [3]-[6], and spatiotemporal light bullets Airy pulses has also being investigated, under linear and nonlinear conditions [7], [8].

Recently, the authors of this study have patented an optical fiber transmission system based on Airy pulses, using the unique properties of these pulses [9]. In this study, we analyze and simulate the proposed transmission system pulses, providing the design tools needed. In the remainder of this study, we analyze the effect of the single-mode fiber (SMF) chromatic dispersion (including cubic-order phase dispersion) on the ideal Airy pulse, as well as a spectrally windowed bandlimited pulse. We also provide the design tools required to obtain the spectral function of a practical finite-energy Airy pulse, by an asymmetrical spectral-domain flat-top windowing of an ideal Airy pulse, which can be used to obtain pulses of different degree of robustness against different amounts of chromatic dispersion. As it is shown, a portion of the temporal waveform keeps invariant, providing a relative insensitiveness of the pulse to variations of chromatic dispersion

\section{Spectral Analysis of Ideal Airy Pulse PROPAGATION ON A SMF}

In the spectral domain, an ideal Airy pulse can be defined from its spectral function as ([10], p. 87)

$$
A(\omega)=\exp \left(j \xi \omega^{3}\right)
$$

where $\omega$ is the baseband angular frequency, i.e., $\omega=$ $\omega_{\text {opt }}-\omega_{0}, \omega_{\text {opt }}$ is the optical angular frequency, $\omega_{0}$ is the central angular frequency, $j$ is the imaginary unit, and $\xi$ is a real constant number. In temporal domain, the corresponding complex envelope function of the Airy pulse, $a(t)$, can be obtained from the inverse Fourier transform of $A(\omega)$. Neglecting the fiber attenuation and nonlinear effects, the spectral response of an SMF section with length $z$ can be modeled as a phase-only filtering responsible of the chromatic dispersion, which can be expressed as $H(\omega, z)=\exp (-j \beta(\omega) z)$, where $\beta(\omega)$ is the propagation constant of the single mode as a function of the baseband angular frequency $\boldsymbol{\omega}$ (centered at $\omega_{0}$ ). The function can be very accurately expressed as the Taylor expansion until third order of $\omega$, leading to the following expression for the transfer function $H(\omega, z)=\exp \left(-j z\left(\beta_{0}+\beta_{1} \omega+\left(\beta_{2} / 2\right) \omega^{2}+\left(\beta_{3} / 6\right) \omega^{3}\right)\right)$, where $\beta_{i}=d^{i} \beta(\omega) / d \omega^{i}$ at $\omega=0$. The quadratic and cubic terms are responsible of the chromatic dispersion, which in general produces a distortion in the complex envelope of the transmitted pulse because of the different values of group velocity of each spectral component. As represented in Fig. 1(a), we can calculate the effect of the Airy pulse propagation thought the fiber as by applying the previous transfer function to the Airy pulse, $F_{\text {prop }}(\omega, z)=A(\omega) H(\omega, z)$, obtaining $F_{\text {prop }}(\omega, z)=$ $\exp \left(-j\left(\beta_{0} z+\beta_{1} z \omega+\left(\beta_{2} z / 2\right) \omega^{2}+\left(\beta_{3} z / 6-\xi\right) \omega^{3}\right)\right)$. It can be demonstrated that the second-order phase term affects as a frequency shift $\Delta \omega(z)$ in the Airy pulse, with

$$
\Delta \omega(z)=\frac{\beta_{2} z}{6 \xi} .
$$

In order to demonstrate this, we can express $F_{\text {prop }}(\omega, z)$ as

$$
\begin{aligned}
& F_{\text {prop }}(\omega, z)= \\
& \quad A(\omega-\Delta \omega(z)) \exp \left(j\left(b_{0}+b_{1} \omega+b_{2} \omega^{2}-\beta_{3} z \omega^{3} / 6\right)\right),
\end{aligned}
$$




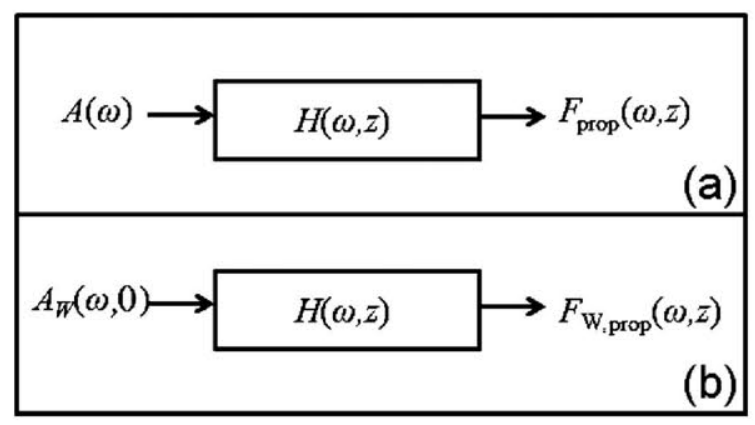

Fig. 1. Spectral input and output signals and transfer function of the optical fiber for (a) ideal Airy pulse as input and (b) bandlimited Airy pulse.

where the coefficients $b_{i}$ can be deduced expanding the Airy pulse expression

$$
\begin{array}{r}
A(\omega-\Delta \omega(z))=\exp \left(j \left(-\xi(\Delta \omega(z))^{3}+3 \xi(\Delta \omega(z))^{2} \omega\right.\right. \\
\left.\left.-3 \xi \Delta \omega(z) \omega^{2}+\xi \omega^{3}\right)\right)
\end{array}
$$

obtaining the coefficients $b_{0}=\xi(\Delta \omega(z))^{3}-\beta_{0} z, b_{1}=$ $-3 \xi(\Delta \omega(z))^{2}-\beta_{1} z$, and the second-order phase term is canceled with $b_{2}=3 \xi \Delta \omega(z)-\beta_{2} z / 2=0$. With all this, we can express

$$
\begin{aligned}
F_{\text {prop }}(\omega, z)=A(\omega-\Delta \omega(z)) & D_{3}(\omega, z) \\
\times & \exp (-j(\Delta t(z) \omega+\phi(z)))
\end{aligned}
$$

where $\phi(z)=-b_{0}=\beta_{0} z-\xi\left(\beta_{2} z / 6 \xi\right)^{3}$ and

$$
\begin{aligned}
\Delta t(z) & =-b_{1}=\beta_{1} z+\frac{\beta_{2}^{2}}{12 \xi} z^{2} \\
D_{3}(\omega, z) & =\exp \left(-j \beta_{3} z \omega^{3} / 6\right) .
\end{aligned}
$$

As shown in the next section, under some conditions, the cubic-order phase distortion produced by $D_{3}(\omega, z)$ can be neglected, and the temporal complex envelope function $f_{\text {prop }}(t, z)$ can be obtained by applying the inverse Fourier transform of (4) as

$$
f_{\text {prop }}(t, z) \approx a(t-\Delta t(z)) \exp (j(\Delta \omega(z) t-\phi(z)))
$$

where $f_{\text {prop }}(t, z)$ is the inverse Fourier transform of $F_{\text {prop }}(\omega, z)$. It can be easily deduced that the waveform invariance of the temporal envelope amplitude is

$$
\left|f_{\text {prop }}(t, z)\right| \approx|a(t-\Delta t(z))| .
$$

Joint time-frequency representations provide a clear visualization of the interaction of pulses with optical media [11], [12]. In this study, we use joint time-frequency representations to visualize the frequency shift and invariant waveform of the Airy pulse during the propagation through the fiber. Fig. 2 shows an illustrative example of the time-frequency distribution of an ideal Airy pulse before and after its propagation through the fiber over a distance, $z$. It can be observed that in both figures, the time-frequency distribution remains undistorted, but there is a frequency shift $\Delta \omega(z)$ of the whole distribution. In time domain, the envelope amplitude remains undistorted. The

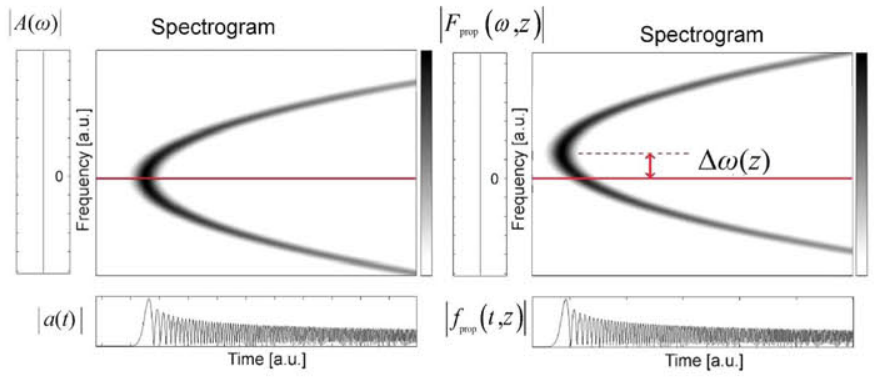

Fig. 2. Frequency shift effect of the dispersion on the time-frequency distribution of an ideal Airy pulse after its propagation in an optical fiber.

cubic-order phase distortion has been neglected in this example. Also, no consideration about the pulse temporal duration and limited bandwidth has been done in this whole section. These aspects are discussed in the next section.

\section{NONIDEAL Airy PUlSE PROPAGATION ON SMF: FLAT-TOP WINDOWED AIRY PULSE}

An ideal Airy pulse has an infinite bandwidth. In a practical implementation, we can only obtain an approximation to the ideal Airy pulse. We can spectrally window the ideal Airy spectral function with a flat-top window function $W(\omega), A_{W}(\omega, 0)=W(\omega) A(\omega)$, where $W(\omega)$ is approximately constant over most of the total window bandwidth, $B$. By using a flat-top window, the resulting spectral function is locally the same as the ideal spectral function, and it can be expected that the invariance property of the pulse will also locally remain. As shown in Fig. 1(b), applying again the transfer function $H(\omega, z)$, and from ideal Airy pulse propagation effect deduced in (4), we can calculate the effect of the fiber dispersion on the bandlimited pulse $A_{\mathrm{W}}(\omega, 0)$

$$
\begin{aligned}
F_{W, \text { prop }}(\omega, z)= & A_{W}(\omega, 0) H(\omega, z) \\
= & W(\omega) A(\omega) H(\omega, z) \\
= & W(\omega) F_{\text {prop }}(\omega, z) \\
= & W(\omega) A(\omega-\Delta \omega(z)) D_{3}(\omega, z) \\
& \times \exp (-j(\Delta t(z) \omega+\phi(z))) \\
= & A_{W}(\omega, z) D_{3}(\omega, z) \\
& \times \exp (-j(\Delta t(z) \omega+\phi(z)))
\end{aligned}
$$

where

$$
A_{W}(\omega, z)=W(\omega) A(\omega-\Delta \omega(z)) .
$$

We provide two conditions to neglect the distortion of the cubic-order phase $D_{3}(\omega, z)$. First, we define a strict nondistortion condition, a sufficient condition to totally neglect the distortion imposing $D_{3}(\omega, z) \approx 1$ in the signal bandwidth $|\omega|<B / 2$, using (6) with $|\omega|=B / 2$

$$
\left|\beta_{3} z B^{3} / 48\right| \ll 2 \pi
$$

This condition has to be met for all $z$ within the path where the pulse invariance is desired. Since it can be too restrictive 
for relatively high bandwidths, we also define a less restrictive condition using the group delay function of $F_{W \text {,prop }}(\omega, z)$

$$
\begin{aligned}
\tau_{W, \text { prop }}(\omega, z) & =-d \angle F_{W, p r o p}(\omega, z) / d \omega \\
& =3\left(\beta_{3} z / 6-\xi\right) \omega^{2}+\beta_{2} z \omega / 2+\beta_{1} z
\end{aligned}
$$

where $\angle$ denotes the phase of the function. From this, we can easily deduce the quasi-nondistortion condition to neglect $\beta_{3}$ in the group delay function $\tau_{W, \text { prop }}(\omega, z)$

$$
\left|\beta_{3} z / 6\right| \ll|\xi| \text {. }
$$

Under this condition, the group delay function of the dispersed pulse is not affected by the cubic phase dispersion term, and it does not distort the overall temporal intensity distribution of the pulse, as shown in the examples. If we neglect the cubic phase term of the dispersion, we can assume $F_{W, \text { prop }}(\omega, z) \approx$ $A_{W}(\omega, z) \exp (-j(\Delta t(z) \omega+\phi(z)))$, and in time domain, we can deduce $f_{\mathrm{W}, \mathrm{prop}}(t, z) \approx a_{\mathrm{W}}(t-\Delta t(z), z) \exp (-j \varphi(z))$, obtaining

$$
\left|f_{W, \text { prop }}(t, z)\right| \approx\left|a_{W}(t-\Delta t(z), z)\right|
$$

where $f_{W, \text { prop }}(t, z)$ and $a_{\mathrm{W}}(t, z)$ are the inverse Fourier transform of $F_{\mathrm{W} \text {,prop }}(\omega, z)$ and $A_{\mathrm{W}}(\omega, z)$, respectively. In order to show the partial invariance of the pulse in the propagation that (14) implies, we proceed to analyze bandlimited, spectrally flat-top windowed Airy pulse, $a_{W}(t, z)$. In Fig. 3, a joint time-frequency analysis of $a_{W}(t, z)$ is shown, where we can observe that the signal can been decomposed in two parts: "head" and "tail.". In the spectral domain, the "head" can be defined as the symmetric spectral section of $A_{\mathrm{W}}(\omega, z)$, which can deduced from Fig. 3 as the spectral portion of $A_{\mathrm{W}}(\omega, z)$ centered in $\Delta \omega(z)$, with bandwidth $B_{\text {head }}(z)$. From (10), we have $A_{\mathrm{W}}(\omega, z) \approx A(\omega-\Delta \omega(z))$ in most of the window bandwidth $B$, and we can finally deduce

$$
F_{\text {head }}(\omega, z) \approx \begin{cases}A(\omega-\Delta \omega(z)), & |\omega-\Delta \omega(z)| \leq B_{\text {head }}(z) \\ 0, & |\omega-\Delta \omega(z)|>B_{\text {head }}(z)\end{cases}
$$

where $B_{\text {head }}(z)=(B-\Delta \omega(z)) / 2$. On the other hand, the "tail" of the pulse can be defined as $F_{\text {tail }}(\omega, z)=A_{W}(\omega, z)-$ $F_{\text {head }}(\omega, z)$. As shown in Fig. 3, the bandlimited Airy temporal envelope function $a_{W}(t, z)$ can be decomposed into

$$
a_{W}(t, z) \begin{cases}\approx f_{\text {head }}(t, z), & t \in I_{\text {head }}(z) \\ \approx f_{\text {tail }}(t, z), & t \in I_{\text {tail }}(z) \\ \approx 0, & t \notin I_{\text {total }}(z)\end{cases}
$$

where $f_{\text {head }}(t, z)$ and $f_{\text {tail }}(t, z)$ are the inverse Fourier transforms of $F_{\text {head }}(\omega, z)$ and $F_{\text {tail }}(\omega, z)$, and $I_{\text {head }}(z), I_{\text {tail }}(z)$, and $I_{\text {total }}(z)$ are, respectively, the time interval corresponding to the "head," "the tail," and the total parts of the pulse.

Since the time-frequency distribution of the "head" of the pulse is locally the same as the frequency-shifted ideal Airy pulse distribution, during the "head" interval of the pulse, the temporal envelope will be approximately the same as the frequency-shifted ideal Airy pulse,

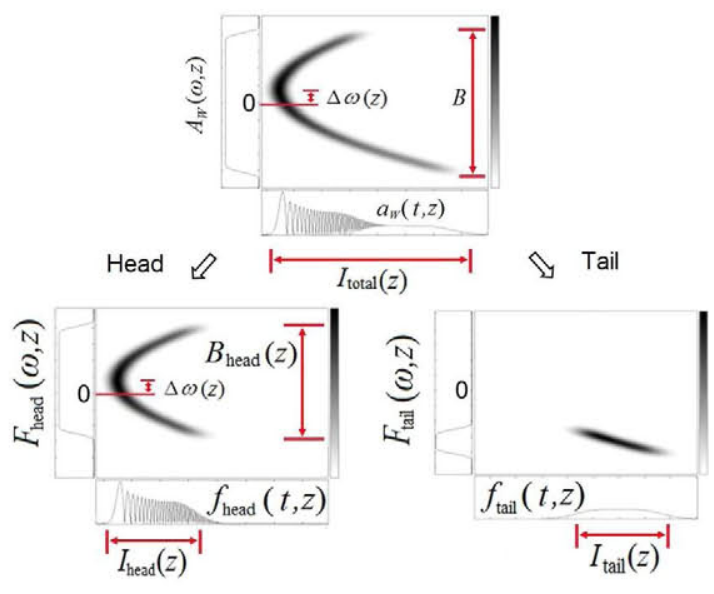

Fig. 3. Time-frequency decomposition of asymmetrically flat-top windowed Airy pulse in "head" and "tail."

$f_{\text {head }}(t, z) \approx a(t) \exp (j \Delta \omega(z) t), t \in I_{\text {head }}(z)$, and the temporal envelope amplitude remains invariant and approximately equal to that of the ideal Airy pulse

$$
\left|f_{\text {head }}(t, z)\right| \approx|a(t)|, \quad t \in I_{\text {head }}(z) .
$$

In order to define the time intervals $I_{\text {total }}(z), I_{\text {head }}(z)$, and $I_{\text {tail }}(z)$, we have to distinguish from the different sign of $\xi$. For $\xi<0$, we can define $I_{\text {total }}(z)=[0, T(z)], I_{\text {head }}(z)=$ $\left[0, T_{\text {head }}(z)\right]$, and $I_{\text {tail }}(z)=\left(T_{\text {head }}(z), T(z)\right]$. In the case $\xi>0$, we have the time reversed version of the signal, and we can define the intervals $I_{\text {total }}(z)=[-T(z), 0], I_{\text {head }}(z)=$ $\left[-T_{\text {head }}(z), 0\right]$, and $I_{\text {tail }}(z)=\left[-T(z),-T_{\text {head }}(z)\right)$. We can approximate the duration of each decomposed part using the Airy pulse group delay function, $\tau_{\mathrm{Aw}}(\omega, z)=-d \angle A_{W}(\omega, z) / d \omega=$ $-3 \xi(\omega-\Delta \omega(z))^{2}$, which is only defined in the signal bandwidth $|\omega|<B$. We can approximate the duration of the whole pulse $T(z)$ as the maximum absolute value of the group delay function, which can be deduced as the maximum absolute value at the edges of the band $\omega= \pm B / 2$

$$
\begin{aligned}
T(z) & =\max \left(\left|\tau_{\mathrm{Aw}}\left(\frac{B}{2}, z\right)\right|,\left|\tau_{\mathrm{Aw}}\left(-\frac{B}{2}, z\right)\right|\right) \\
& =3|\xi|\left(\frac{B}{2}+|\Delta \omega(z)|\right)^{2} .
\end{aligned}
$$

Also, we can approximate the duration of the "head" of the pulse as the maximum absolute value of the group delay function in the spectral range of the "head," which can be deduced as the minimum absolute value at the edges of the band $\omega= \pm B / 2$

$$
\begin{aligned}
T_{\text {head }}(z) & =\min \left(\left|\tau_{\text {Aw }}\left(\frac{B}{2}, z\right)\right|,\left|\tau_{\text {Aw }}\left(-\frac{B}{2}, z\right)\right|\right) \\
& =3|\xi|\left(\frac{B}{2}-|\Delta \omega(z)|\right)^{2} .
\end{aligned}
$$

\section{PRoposed DESIGN}

In our proposed design, we consider a situation represented in Fig. 4, with an invariant path with length $L$, and a preinvariant path with length $l$, where $z$ origin $(z=0)$ is set in the center of the invariant path. The preinvariant path is defined to consider 


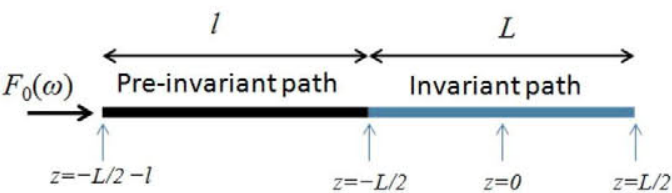

Fig. 4. Representation of input signal and optical fiber paths considered in the proposed design.

the possibility of introducing the pulse at an arbitrary distance $l$ before the invariant path. The objective of the design consists in obtaining the expression of the pulse at the beginning of the preinvariant path to be introduced in the fiber at $z=-L / 2-l$, which lead to a pulse with a spectral function $A_{W}(\omega, 0)$ in the center of the invariant path, at $z=0$. We can define this initial pulse by using an spectral function $F_{0}(\omega)$. In the previous section, we have obtained the expression of a pulse at an arbitrary $z$, assuming a pulse $A_{W}(\omega)$ at a position $z=0$. The previous expressions can also be used for negative values of $z$. Therefore, the corresponding pulse at $z=-L / 2-l$ can be obtained using (9). If we neglect linear and constant phase term (which does not affect to the pulse waveform), we obtain $F_{0}(\omega)=A_{W}(\omega,-L / 2-l) D_{3}(\omega,-L / 2-l)$, and using (6) and (10), it can be expressed as

$$
\begin{aligned}
F_{0}(\omega)=W(\omega) \exp \left(j\left(\xi-\beta_{3}(-L / 2-l)\right)\right. \\
\left.\times\left(\omega-\beta_{2}(L / 2+l) / 6 \xi\right)^{3}\right) .
\end{aligned}
$$

The condition for strict nondistortion of the pulse due to cubic phase during the invariant path can be obtained from (11) using the extreme values $|\omega|=B / 2$ and $|z|=L / 2$

$$
\left|\beta_{3} L B^{3} / 96\right| \ll 2 \pi
$$

and the condition for quasi-nondistortion of the pulse due to cubic phase can be obtained from (13) using $|z|=L / 2$

$$
\left|\beta_{3}(L / 12)\right| \ll|\xi|
$$

Let us define some interesting design parameters in the desired invariant path $z \in[-L / 2, L / 2]$. The maximum absolute deviation of the pulse frequency $\Delta \omega_{\max }$ regarding the central frequency, in the extreme points of the path, $z= \pm L / 2$ can be obtained from

$$
\begin{aligned}
\Delta \omega_{\max } & =\max (|\Delta \omega(z)|) \\
& =|\Delta \omega(L / 2)|=|\Delta \omega(-L / 2)|=\left|\frac{\beta_{2} L}{12 \xi}\right| .
\end{aligned}
$$

We also define two temporal parameters, $T_{\mathrm{inv}}$ and $T_{\max }$, where $T_{\mathrm{inv}}$ is the invariant part of the pulse duration in the whole path $z \in[-L / 2, L / 2]$, and can be calculated as the minimum duration of the head of pulse, and $T_{\max }$ is the maximum total duration of the pulse in the whole path. These extreme

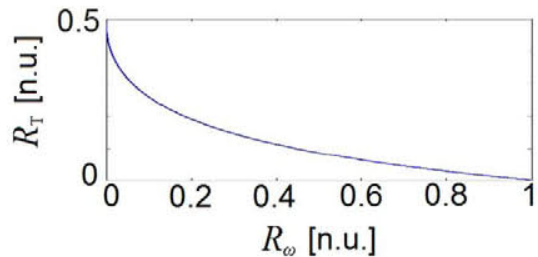

Fig. 5. Relation between $R_{T}$ and $R_{\omega}$.

values occur in the extreme points of the path, $\mathrm{z}= \pm L / 2$. From (18) and (19), we can deduce

$$
\begin{aligned}
& T_{\text {inv }}=\min \left(T_{\text {head }}(z)\right)=3|\xi|\left(\frac{B}{2}-\Delta \omega_{\max }\right)^{2} \\
& T_{\max }=\max (T(z))=3|\xi|\left(\frac{B}{2}+\Delta \omega_{\max }\right)^{2} .
\end{aligned}
$$

Let us also define a temporal ratio $R_{T}=T_{\mathrm{inv}} / T_{\max } \in(0,1)$, which indicates "how invariant" is the temporal amplitude envelope of our spectrally windowed Airy pulse as it propagates through the fiber, obtained from the proportion between $T_{\mathrm{inv}}$ and $T_{\max }$. We also define the spectral ration $R_{\omega}=\Delta \omega_{\max } / B \in$ $(0,1 / 2)$, which indicates the maximum relative frequency variation of the pulse. Both parameters $R_{T}$ and $R_{\omega}$ can be obtained as

$$
\begin{aligned}
& R_{T}=T_{\text {inv }} / T_{\max }=\frac{\left(B-2 \Delta \omega_{\max }\right)^{2}}{\left(B+2 \Delta \omega_{\max }\right)^{2}}=\frac{\left(1-2 R_{\omega}\right)^{2}}{\left(1+2 R_{\omega}\right)^{2}} \\
& R_{\omega}=\Delta \omega_{\max } / B=\frac{1+R_{T}-2 \sqrt{R_{T}}}{2-2 R_{T}} .
\end{aligned}
$$

In Fig. 5, $R_{T}$ and $\boldsymbol{R}_{\boldsymbol{\omega}}$ are represented, where it can be observed that theoretically we can obtain a perfectly invariant pulse, with $R_{T} \approx 1$ and $R_{\omega} \approx 0$.

\section{Examples AND Simulation Results}

Without loss of generality, in these examples, we are going to assume standard SMF (ITU-T G.652) in which dispersion parameter can be modeled as $D(\lambda)=D_{1550}+S_{1550}\left(\lambda-\lambda_{0}\right)$ $\mathrm{ps} /(\mathrm{nm} \cdot \mathrm{km})$, where $\lambda_{0}=1550 \mathrm{~nm}, D_{1550}=17 \mathrm{ps} /(\mathrm{nm} \cdot \mathrm{km})$, and $S_{1550}=0.056 \mathrm{ps} /\left(\mathrm{nm}^{2} \cdot \mathrm{km}\right)$. From this, we can obtain $\beta_{2}=-21.68 \mathrm{ps}^{2} / \mathrm{km}$ and $\beta_{3}=0.0911 \mathrm{ps}^{3} / \mathrm{km}$ at the central frequency $\omega_{0}=2 \pi f_{0}$ with $f_{0}=193.413 \mathrm{THz}$. Nonlinear effects are out of the scope of this paper, and fiber attenuation will not be considered in order to have a more clear visualization of the pulse waveform invariance.

As a first example, we consider a total signal bandwidth of 0.1 THz, i.e., $B=B_{1}=2 \pi \cdot 0.1 \mathrm{THz} \cdot \mathrm{rad}$ centered at $\omega_{\mathrm{opt}}=\omega_{0}$, and a desired invariant path length of $L=100 \mathrm{~km}$, without preinvariant path, $l=0$, where the initial pulse is supposed to be introduced right at the beginning of the invariant path. From the strict nondistortion condition of (21), we obtain a phase value of $0.0238 \mathrm{rad}$, which can be neglected regarding $2 \pi$. The strict nondistortion condition is a sufficient condition for completely neglecting the cubic phase propagation distortion. Using 


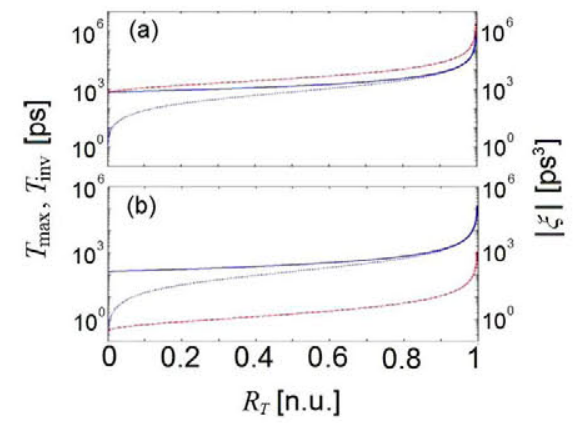

Fig. 6. Representation of $T_{\max }$ (blue solid), $T_{\mathrm{inv}}$ (blue dotted), and $|\xi|$ (red dotted) as a function of $R_{T}$ for (a) first and (b) second examples.

(24)-(27) and (2), we can calculate $T_{\max }, T_{\mathrm{inv}}$, and $\xi$ parameters as a function of $R_{T}$ ratio, as shown in Fig. 6(a). In principle, we can theoretically obtain a value of $R_{T}$ as close to 1 as desired, but $T_{\max }, T_{\mathrm{inv}}$, and $\xi$ asymptotically tend to infinity when $R_{T}=1$. Therefore, it is theoretically possible to get as degree of invariance as close to the perfect invariance as desired, although it may lead to impractical designs as we approach to the ideal perfect invariance.

For illustrative purposes, we select a medium value of $R_{T}=$ 0.5 , which lead to the values $T_{\max }=1.36 \mathrm{~ns}, T_{\mathrm{inv}}=0.681 \mathrm{~ns}$, and $\xi=3.351 \cdot 10^{3} \mathrm{ps}^{3}$, and from (27), we obtain a maximum relative deviation of frequency $R_{\omega}=0.0858$ and $\Delta \omega_{\max }=$ $R_{\omega} \cdot B=2 \pi \cdot 8.58 \mathrm{GHz} \cdot \mathrm{rad}$. The initial pulse to be introduced in the fiber at $z=-L / 2$ is spectrally defined by (20). For the window function $W(\omega)$, we have selected a raised cosine function with a roll-off factor of $20 \%$, with a total bandwidth $\boldsymbol{B}$. Fig. 7 shows the temporal amplitude envelope, as well as the joint time-frequency analysis, of the resulting propagated pulse obtained from numerical simulation for three positions of the invariant path $(z=-L / 2,0$, and $L / 2)$. Because of the positive value of $\boldsymbol{\xi}$, the pulse is reversed from the pulses shown previously, where negative values of $\xi$ were assumed. The selected value of $R_{T}=0.5$ implies $T_{\mathrm{inv}}=T_{\max } / 2$, and therefore, the 'head' of the pulse has approximately same duration as the "tail" in the extremes of the path, $z= \pm L / 2$. In the center of the invariant path $z=0$, there is no tail and the "head" duration is equal to the total duration of the pulse, $T_{\text {head }}(0)=T(0)$.

As a second example, we consider a signal bandwidth of 2 THz, i.e., $B=2 \pi \cdot 2 \mathrm{THz} \cdot \mathrm{rad}$ centered at $\omega_{\text {opt }}=\omega_{0}$, a desired invariance path duration of $L=1 \mathrm{~km}$, and a preinvariant path of $l=3 \mathrm{~km}$. Applying (21) for the cubic phase strict nondistortion condition, we get a phase value of $1.88 \mathrm{rad}$, which can obviously not be neglected regarding $2 \pi$. Even if the strict nondistortion condition is not satisfied, we still can get little cubic phase distortion if the quasi-nondistortion condition is satisfied; from (22), $\xi \gg 0.0152 \mathrm{ps}^{3}$. In Fig. 6(b), we represent again $T_{\max }, T_{\mathrm{inv}}$, and $\xi$ parameters as a function of $R_{T}$. We select $R_{T}=0.7$, which leads to $T_{\max }=0.454$ $\mathrm{nm}, T_{\mathrm{inv}}=0.318 \mathrm{~nm}$, and a value of $\xi=-3.233 \mathrm{ps}^{3}$ that satisfies the quasi-nondistortion condition. The negative value of $\xi$ is selected to illustrate the inverted temporal waveform of the pulse regarding the previous example. We also obtain $R_{\omega}=0.0445, \Delta \omega_{\max }=2 \pi \cdot 0.89 \mathrm{THz} \cdot \mathrm{rad}$. The initial pulse at $z=-l-L / 2$ to be generated and introduced in the fiber

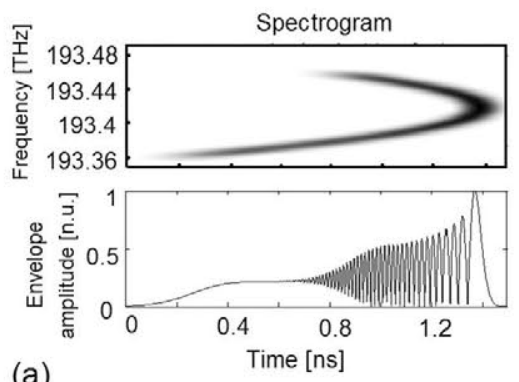

(a)

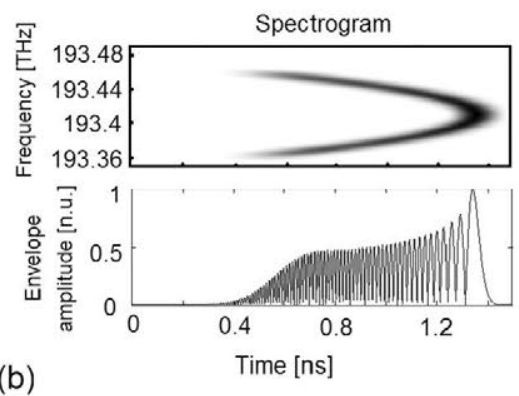

(b)
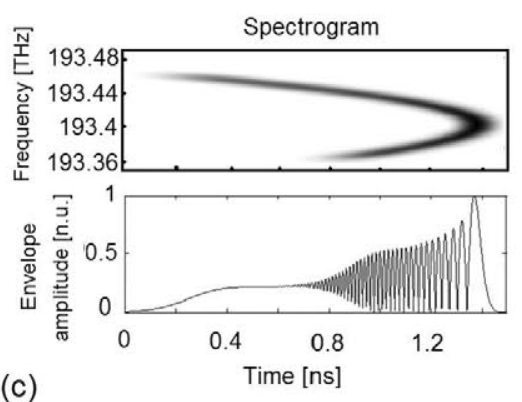

Fig. 7. Invariant path simulation results for the first example. Temporal envelope amplitude and joint time-frequency analysis. (Multimedia file included.) (a) $z=-50 \mathrm{~km}$. (b) $z=0 \mathrm{~km}$. (c) $z=50 \mathrm{~km}$.

is spectrally defined in (20). Again, a raised cosine function is used as a window function $W(\omega)$, with a roll-off factor of $20 \%$ and a total bandwidth $B$. Fig. 8 shows the temporal amplitude envelope, as well as the joint time-frequency analysis, of the resulting propagated pulse, obtained for three positions of the invariant path $(z=-L / 2,0$, and $L / 2)$. The selected value of $R_{T}=0.7$ implies $T_{\mathrm{inv}}=0.7 \cdot T_{\max }$

In order to better illustrate the pulse propagation through the fiber in the invariant path, we have included an animation for each example. We have included two supplementary AVI files that show a representation of the pulse propagation in the invariant path, for first and second example, respectively, corresponding to intermediate $z$ values of the invariant path. We can observe the invariance of the "head" of the pulse, with a minimum duration of the invariant part $T_{\mathrm{inv}}$ and a maximum total duration $T_{\max }$, as deduced theoretically. It is also interesting to observe the nonuniform velocity effect (acceleration) of the pulse. As indicated in (5), the dependence of the delay of the pulse $\Delta t(z)$ on $z$ is not linear, and the pulse velocity is not constant. This nonuniform velocity is due to the fact that the group velocity function depends on frequency, and therefore, the frequency shift $\Delta \omega(z)$ also produces a variation in the pulse apparent velocity. We can express $\Delta t(z)=\Delta t_{1}(z)+$ $\Delta t_{2}(z)$, where $\Delta t_{1}(z)=\beta_{1} z$ and $\Delta t_{2}(z) \approx \beta_{2}^{2} z^{2} /(12 \xi)$. The 


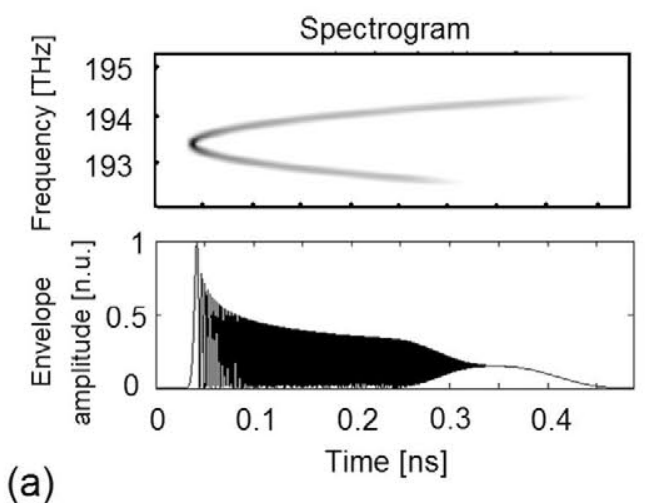

(a)

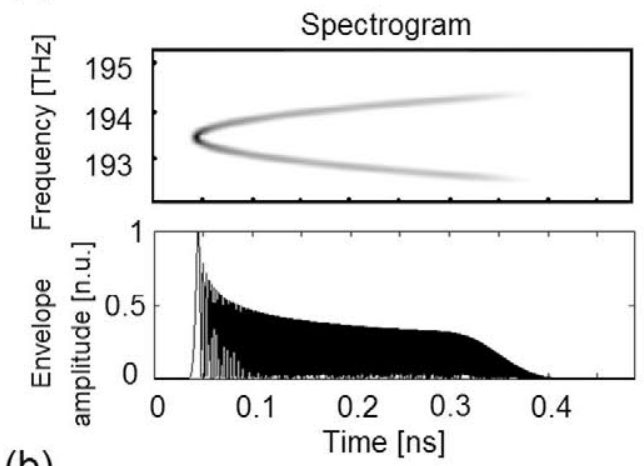

(b)
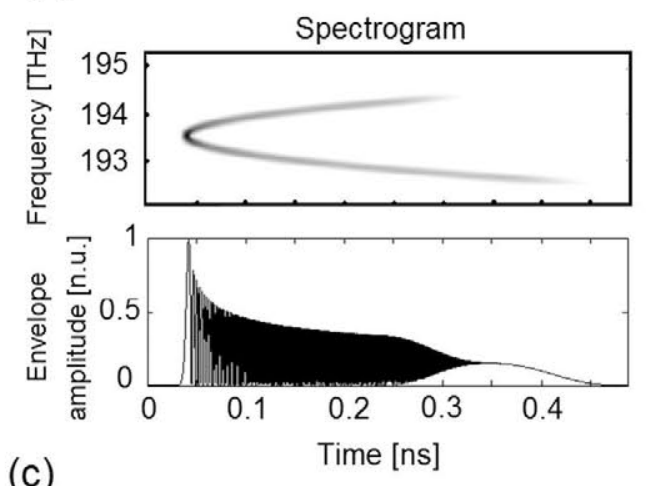

(c)

Fig. 8. Invariant path simulation results for the second example. Temporal envelope amplitude and joint time -frequency analysis. (Multimedia file included.) (a) $z=-0.5 \mathrm{~km}$. (b) $z=0 \mathrm{~km}$. (c) $z=0.5 \mathrm{~km}$.

delay term $\Delta t_{1}(z)$ constituting the main delay component of the pulse in its propagation through the fiber is subtracted from the total pulse delay represented. Thus, the pulse delay observed in the animations only corresponds to $\Delta t_{2}(z)$ term. The maximum delay variation in the invariant path due to $\Delta t_{2}(z)$ term is $\Delta t_{2}(L / 2)=\Delta t_{2}(-L / 2)=29.21 \mathrm{ps}$ for the first example, and $\Delta t_{2}(L / 2)=\Delta t_{2}(-L / 2)=-3.03 \mathrm{ps}$ for the second example. Therefore, it is a very marginal effect compared to the main delay component $\Delta t_{1}(z)$, which is in the order of microseconds for distances in the order of kilometers.

Fig. 9 represents the initial pulse to be generated at the beginning of the preinvariant path for the second example (it was assumed no preinvariant path in the first example), and we have also included a supplementary AVI file that shows the animation of the propagation of the pulse during the preinvariant path. As can be observed in the animation, as the pulse propagates in the fiber, its waveform evolves to the desired pulse in the invariant

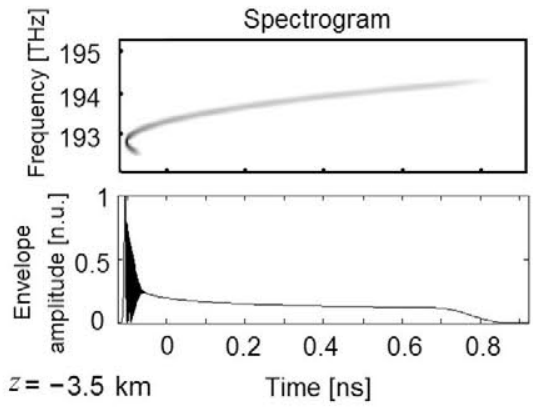

Fig. 9. Preinvariant path simulation results for the second example. Temporal envelope amplitude and joint time-frequency analysis of the pulse. (Multimedia file included.)
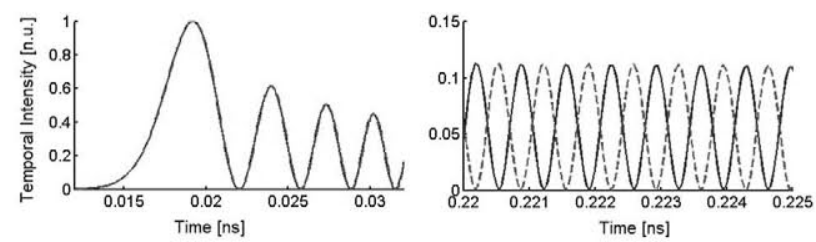

Fig. 10. Cubic phase distortion for the second example, comparing the propagating pulse at the initial point of the invariant path $z=-L / 2$ (red dotted) and the final point of the invariant path $z=L / 2$ (blue solid).

path, as predicted in the previous theory. Nonuniform velocity effect can also be observed, where again the main propagation delay term, $\Delta t_{1}(z)$, is subtracted from the total pulse delay.

In Fig. 10, we show how the cubic phase term distortion affects to the pulse temporal waveform in the second example (for the first example, the cubic phase distortion is neglectable), where the whole delay term $\Delta t$ has been subtracted for a clear visualization. As can be observed, it causes some shift in the ripples of the pulse, but the overall envelope shape remains undistorted, verifying the validity of the proposed quasi-nondistortion condition.

It is also worth noting that, since only linear effects are involved in the invariant propagation of Airy pulses, a perturbation or noise added to the initial Airy pulse affects similarly as in generic pulses propagation under linear conditions, being linearly summed to the predicted invariant propagated pulse.

\section{CONCLUSION}

Here, we have extensively developed the analytic basis for design invariant bandlimited Airy pulses in SMFs, using the unique propagation properties of Airy pulses in dispersive media. Joint time-frequency analysis plays an important role in this analysis and design. In order to get the finite energy Airy pulse, a truncation in the spectral domain (instead of temporal domain like in [2]) is applied. This spectral truncation consists in applying an asymmetric flat-top window to the ideal Airy pulse spectra, which does not locally affect the unique properties of the Airy beams.

There are several pulse shaping techniques that can be applied to generate the initial pulse of the proposed designs. Fiber Bragg gratings (FBGs) [13] and spatial light modulators [14] have shown to be very suitable in shaping optical pulses of relative complexity. FBG-based implementations are an in-fiber 
inexpensive solution, which have been demonstrated for introducing high amounts of pure third-order dispersion [15]. Spatial light modulators have been implemented in commercial highly sophisticated pulse shapers devices, which can be used for this purpose in a more flexible and reconfigurable way, but with higher costs.

Regarding the potential use of Airy pulses in fiber-optic communication systems, from the previous analysis and examples, it can be observed that very long pulses and very large pulse bandwidth are required. This, in principle, can imply a serious limitation of such a system because of the little spectral efficiency, unless a controlled intersymbol interference scheme based on the propagation properties of these pulses is applied.

As we have shown, we can design a finite Airy pulse with a defined degree of invariance to chromatic dispersion. It is theoretically possible to obtain an arbitrarily high degree of invariance, although a too high required degree of invariance may lead to impractical designs. Therefore, we need to obtain a tradeoff solution between the degree of invariance and the feasibility of the desired initial pulse.

\section{REFERENCES}

[1] M. V. Berry and N. L. Balazs, "Nonspreading wave packets," Amer. J. Phys., vol. 47, pp. 264-267, 1979.

[2] G. Siviloglou and D. N. Christodoulides, "Accelerating finite energy Airy beams," Opt. Lett., vol. 32, pp. 979-981, 2007.

[3] G. Siviloglou, J. Brokey, A. Dogariu, and D. N. Christodoulides, "Observation of accelerating Airy beams," Phys. Rev. Lett., vol. 99, pp. 213901-1-213901-4, 2007.

[4] J. Baumgartl, M. Mazilu, and K. Dholakia, "Optically mediated particle clearing using Airy wavepackets," Nature Photon., vol. 2, pp. 675-678, 2008.

[5] P. Polynkin, M. Kolesik, J. V. Moloney, G. A. Siviloglou, and D. N. Christodoulides, "Curved plasma channel generation using ultraintense Airy beams," Science, vol. 324, pp. 229-232, 2009.
[6] I. Dolev, T. Ellenbogen, and A. Arie, "Switching the acceleration direction of Airy beams by a nonlinear optical process," Opt. Lett., vol 35, pp. 1581-1583, 2010.

[7] A. Chong, W. H. Renninger, D. N. Christodoulides, and F. W. Wise, "Airy-Bessel wave packets as versatile linear light bullets," Nature Photon., vol. 4, pp. 103-106, 2010.

[8] D. Abdollahpour, S. Suntsov, D. G. Papazoglou, and S. Tzortzakis, "Spatiotemporal Airy light bullets in the linear and nonlinear regimes," Phys. Rev. Lett., vol. 105, pp. 253901-1-253901-4, 2010.

[9] M. A. Preciado and M. A. Muriel, "," Spain Patent Es2364935, 2010.

[10] O. Vallée and M. Soares, Airy Functions and Applications to Physics. London, U.K.: Imperial College Press, 2004.

[11] J. Azaña and M. Muriel, "Study of optical pulses-fiber gratings interaction by means of joint time-frequency signal representations," $J$. Lightw. Technol., vol. 21, no. 11, pp. 2931-2941, Nov. 2003.

[12] J. Azaña, "Time-frequency (Wigner) analysis of linear and nonlinear pulse propagation in optical fibers," EURASIP J. Appl. Signal Process. vol. 2005, no. 10, pp. 1554-1565, 2005.

[13] P. Petropoulos, M. Ibsen, A. D. Ellis, and D. J. Richardson, "Rectangular pulse generation based on pulse reshaping using a superstructured fiber Bragg grating," J. Lightw. Technol., vol. 19, no. 5, pp. 746-752, May 2001.

[14] A. M. Weiner, "Femtosecond optical pulse shaping and processing," Prog. Quantum Electron., vol. 19, pp. 161-235, 1995.

[15] M. Ibsen and R. Feced, "Fiber Bragg gratings for pure dispersion-slope compensation," Opt. Lett., vol. 28, pp. 980-982, 2003.

Miguel A. Preciado received the Ph.D. degree in electrical engineering from the Universidad Politécnica de Madrid, Madrid, Spain, in 2010

In 2011, he joined the Aston Institute of Photonic Technologies, Aston University, Birmingham, U.K.

Dr. Preciado is a member of the Optical Society of America. He is the recipient of a Marie Curie Intra-European Fellowship in 2011.

Miguel A. Muriel (SM'98) received the Ph.D. degree in electrical engineering from the Universidad Politecnica de Madrid (UPM), Madrid, Spain, in 1980.

From 1989 to 1997, he was the Head of the Department of Photonics Technology, UPM, where since 1989, he has been a Full Professor. He has contributed to more than 165 international papers and conference contributions in photonic signal processing. He has been an advisor of $13 \mathrm{Ph}$.D. students, and has led 14 research engineering projects.

Dr. Muriel is a senior member of the Optical Society of America. 